\title{
The NK-1 receptor is expressed in human melanoma and is involved in the antitumor action of the NK-1 receptor antagonist aprepitant on melanoma cell lines
}

\author{
Miguel Muñoz ${ }^{1}$, Marisa Rosso ${ }^{1}$, María José Robles-Frias², Manuel Vicente Salinas-Martín², Rosario Rosso ${ }^{1}$, \\ Ana González-Ortega ${ }^{1}$ and Rafael Coveñas ${ }^{3}$
}

\begin{abstract}
Melanoma, the most deadly form of skin cancer, is aggressive and resistant to current therapies. It has been previously reported that the substance P and neurokinin-1 (NK-1) receptor antagonists induce cell proliferation and cell inhibition, respectively, in human melanoma cell lines. Aprepitant is a selective high-affinity antagonist of the human NK-1 receptor. Until now, this drug has been used as an anxiolytic, antidepressant and antiemetic. Moreover, the antitumor action of aprepitant has been previously reported. However, the presence of NK-1 receptors in human melanomas and whether the antitumor action of the NK-1 receptor antagonist aprepitant is exerted on human malignant melanomas have not been previously described. The aims of this study are to show the presence of NK-1 receptors in human malignant melanomas and the antitumoral action of aprepitant against several human melanoma cell lines. Immunoblot analysis was used to determine the presence of NK-1 receptors in human melanoma cell lines, and immunohistochemistry was used to demonstrate NK-1 receptors in human melanoma samples. We performed an in vitro study of the cytotoxicity of the NK-1 receptor antagonist aprepitant on human melanoma cell lines. A coulter counter was used to determine viable cell numbers, followed by application of the tetrazolium compound MTS. The DAPI method was applied to demonstrate apoptosis. We observed that NK-1 receptors were present in all the melanoma samples studied as well as in human melanoma cell lines. We also showed that melanoma cell lines expressed mRNA for the NK-1 receptor. Moreover, after using a knockdown method, we showed that NK-1 receptors are involved in the viability of tumor cells. In this study, we also report that aprepitant, at 10-60 $\mathrm{M}$ concentrations, elicits cell growth inhibition in a concentration-dependent manner in all melanoma cell lines studied, that the specific antitumor action of aprepitant occurs through the NK-1 receptor and that melanoma cell death is due to apoptosis. These findings show for the first time that the NK-1 receptor may be a promising new target and that the NK-1 receptor antagonist aprepitant could be a candidate as a new antitumor drug in the treatment of human melanoma.
\end{abstract}

Laboratory Investigation (2010) 90, 1259-1269; doi:10.1038/labinvest.2010.92; published online 10 May 2010

KEYWORDS: antitumor; aprepitant; drug; melanoma; NK-1 receptor

Melanoma is a neoplasm originating from melanocytes, which are derived from the neural crest. Melanoma now accounts for $\sim 4 \%$ of all cancers diagnosed in the United States, and there were $\sim 8110$ deaths from the disease in 2007. ${ }^{1}$ The last two decades have seen no significant progress in extending the survival of patients with distant metastases, despite multiple trials of cytotoxic chemotherapeutic agents.
There is thus an urgent need to improve therapy in patients with melanoma.

Substance P (SP) is an undecapeptide that belongs to the tachykinin family of peptides. It is known that SP, neurokinin A (NKA), neuropeptide $K$ and neuropeptide- $\gamma$ (the latter two being elongated forms of NKA) are derived from the preprotachykinin A gene, whereas neurokinin $B(\mathrm{NKB})$ is

\footnotetext{
${ }^{1}$ Research Laboratory on Neuropeptides, Virgen del Rocío University Hospital, Sevilla, Spain; ${ }^{2}$ Department of Pathology, Virgen del Rocío University Hospital, Sevilla, Spain and ${ }^{3}$ Laboratory of Neuroanatomy of the Peptidergic Systems, Institute of Neurosciences of Castilla y León (INCYL, Lab. 14), Salamanca, Spain Correspondence: Dr M Muñoz, PhD, Hospital Infantil Virgen del Rocío, Unidad de Cuidados Intensivos Pediátricos, Av. Manuel Siurot s/n, 41013, Sevilla, Spain. E-mail: mmunoz@cica.es

Received 3 March 2009; revised 26 February 2010; accepted 26 March 2010
} 
derived from the preprotachykinin $B$ gene. The biological actions of SP, NKA and NKB are mediated by three receptors, namely neurokinin (NK)-1, NK-2 and NK-3, the NK-1 receptor showing preferential affinity for SP. After binding to the NK-1 receptor, SP regulates many biological functions. ${ }^{2}$ This neuropeptide has been implicated in neurogenic inflammation, pain and depression, ${ }^{3}$ as well as in tumor cell proliferation, neoangiogenesis and metastasis. ${ }^{4}$ In addition, the expression of SP has been reported in primary invasive malignant melanomas, metastatic melanomas, in situ melanomas, atypical (dysplastic) nevi, as well as spindle and epithelioid cell (Spitz) nevi, but was not detected in any acquired benign melanocytic nevi. ${ }^{5}$ It has also been shown that activation of NK-1 receptors by SP induces mitogenesis in several melanoma cell lines ${ }^{6,7}$ and others tumor cell lines. ${ }^{8-16}$ $\mathrm{SP}$ is also a main mediator in the growth of capillary vessels in vivo and in the proliferation of cultured endothelial cells in vitro, and it has also been shown that NK-1 receptor agonists induced neoangiogenesis. ${ }^{17}$ Moreover, the active migration of tumor cells, a crucial requirement for invasion and metastasis, is regulated by SP signals. ${ }^{18}$

NK-1 receptors have been localized and characterized in several human neoplasms. ${ }^{4,7,16,19-24}$ Moreover, the expression of NK-1 receptors has also been reported in human glioma, neuroblastoma, pancreatic carcinoma, retinoblastoma, laryngeal carcinoma, as well as in gastric and colon carcinoma cell lines. ${ }^{10,12,15,16,21,24}$

The NK-1 receptor antagonist L-733060 (a piperidine derivative) shows a high affinity for the human NK-1 receptor in vitro. ${ }^{25}$ Moreover, we have previously shown that L-733060 shows antitumor activity against human malignant melanoma cell lines ${ }^{6}$ and neuroblastoma, glioma, retinoblastoma, pancreas, larynx, gastric and colon carcinoma cell lines. ${ }^{11,12,15,16,26}$ Aprepitant is a selective high-affinity antagonist of the human NK-1 receptor. Until now, this drug has been used as an antidepressant, anxiolytic and antiemetic. ${ }^{27-29}$ Despite this, we have recently reported that aprepitant is a broad-spectrum antitumor drug. ${ }^{30}$ However, to our knowledge, the presence of NK-1 receptors in melanomas and whether the antitumor action of the NK-1 receptor antagonist aprepitant is exerted on human malignant melanomas are unknown. Thus, the aims of this study are: (1) to demonstrate in vitro the presence of NK-1 receptors in MEL HO, COLO 858 and COLO 679 human melanoma cell lines; (2) to demonstrate the presence and distribution of the NK-1 receptor in several human primary invasive malignant melanomas and metastatic melanomas; (3) to demonstrate that melanoma cell lines express mRNA for the NK-1 receptor; (4) to evaluate the involvement of NK-1 receptors on the viability of tumor cells; (5) to demonstrate, using an MTS colorimetric method to evaluate cell viability, the antitumor action of the NK-1 receptor antagonist aprepitant against human melanoma cell lines, and to show that this antitumor action occurs through the NK-1 receptor; and (6) to determine whether the NK-1 receptor antagonist aprepitant causes apoptosis in the melanoma cell lines studied.

\section{MATERIALS AND METHODS \\ Cell Culture}

We used COLO 858 (ICLC Interlab Cell Line CollectionCBA-Genova), MEL HO and COLO 679 human melanoma cell lines (DSMZ-Deutsche Sammlung von Mikroorganismen und Zellkulturen). These cell lines were incubated at $37^{\circ} \mathrm{C}$ in a humidified atmosphere of $95 \%$ air $/ 5 \% \mathrm{CO}_{2}$ according to the manufacturer's instruction.

\section{Drug Treatments}

The NK-1 receptor antagonist 5-[[(2R, 3S)-2-[(1R)-1-[3,5bis(trifluoromethyl) phenyl] ethoxy]-3-(4-fluorophenyl)-4morpholinyl]methyl]-1,2-dihydro-3 H-1,2,4-triazol-3-one (aprepitant: MW 534.43) (Merck Research Laboratories, Madrid, Spain) was dissolved in distilled water containing acetonitrile before sample treatment. To determine $\mathrm{IC}_{50}$, different concentrations $(10-60 \mu \mathrm{M})$ of aprepitant were evaluated. SP, acetate salt (Sigma-Aldrich, Madrid, Spain), was dissolved in distilled water and different concentrations $(5,10,50,100$ and $500 \mathrm{nM})$ were used. The most mitogenic nanomolar SP concentration for each cell line was incubated for $1 \mathrm{~h}$ before the addition of aprepitant.

\section{Proliferation Assays}

Cell proliferation was evaluated using the tetrazolium compound 3-(4, 5-dimethylthiazol-2-yl)-5-(3-carboxymethoxyphenyl)2-(4-sulfophenyl)-2H-tetrazolium, inner salt (MTS), according to the manufacturer's instructions (CellTiter 96 Aqueous One-Solution Cell Proliferation Assay, Promega, Madison, USA). Cell numbers were quantified using a Coulter counter. The plate included blank wells $(0$ cells/ $0.1 \mathrm{ml})$, control wells $\left(10^{4}\right.$ cells $\left./ 0.1 \mathrm{ml}\right)$, control wells with acetonitrile, control wells treated with aprepitant and control wells treated with the most effective SP concentration and aprepitant. The plates were inoculated with aprepitant (10-60 $\mu \mathrm{M}$ for tumor cell lines) and were incubated for the first doubling time specific for each tumor cell line. Plates were also inoculated with the most mitogenic exogenous SP nanomolar concentration with or without the $50 \% \mu \mathrm{M}$ inhibition concentration $\left(\mathrm{IC}_{50}\right)$ of aprepitant for their first doubling times. Moreover, plates were also inoculated with increasing concentrations of aprepitant $(30,40,50$ and $60 \mu \mathrm{M})$ and for each concentration of the drug, gradual increasing concentrations of SP were added (10, 50, 100 and $500 \mathrm{nM}$ ). For the proliferation assay, $20 \mu \mathrm{l}$ of the MTS reagent was added to each well $90 \mathrm{~min}$ before reading the samples on a multiscanner microplate reader (TECAN Spectra classic, Barcelona, Spain) at $492 \mathrm{~nm}$. Each experimental condition was assayed in duplicate and all experiments were performed at least three times. 


\section{Statistical Analyses}

Data were expressed as means \pm s.d. Statistical analyses were performed using the SPSS statistical software for Microsoft Windows, release 13.0 (Professional Statistic, Chicago, IL, USA). The homogeneity of variance was tested using the Levene test. If the variances were homogeneous, data were analyzed using the one-way ANOVA test with Bonferroni's correction for multiple comparisons. For data sets with nonhomogeneous variances, the ANOVA test with T3 Dunnett's post hoc analysis was applied. The criterion for significance was $P<0.05$ for all comparisons.

\section{DAPI Staining}

To determine whether apoptosis was induced by the NK-1 receptor antagonist aprepitant, DAPI staining was performed. Briefly, after treatment with aprepitant for their approximately first doubling times or after the application of the knockdown method (see below), the cells were fixed in $4 \%$ paraformaldehyde. After a second wash in PBS, cells were incubated in DAPI solution (Sigma-Aldrich) at a dilution of $1 / 1000(=1 \mu \mathrm{g} / \mathrm{ml})$ for $30 \mathrm{~min}$ in the dark. Cells were then observed through a fluorescence microscope (Zeiss, Oberkochen, Germany). Apoptotic cells were defined by chromatin condensation and nuclear fragmentation. We counted the number of apoptotic cells, repeating the counts on three different slides. Finally, on each slide, we counted the number of apoptotic cells located in five different sequential fields.

\section{Western Blot Analyses}

As previously reported, ${ }^{10}$ total protein was prepared from subconfluent MEL HO, COLO 858 and COLO 679 human melanoma cell cultures. Protein concentrations were determined using the protein assay kit purchased from Bio-Rad according to the manufacturer's instructions.

From each sample, $50 \mu \mathrm{g}$ of protein was separated by electrophoresis on 10\% SDS-polyacrylamide gels and electroblotted onto PVDF membranes. Blots were incubated in blocking solution (5\% nonfat milk in PBS, $0.1 \%$ Tween-20 (PBS-T)), followed by overnight incubation with an antibody against the KTMTESSSFYSNMLA conserved domain, corresponding to the $\mathrm{C}$ terminus of the NK-1 receptor (Product number S8305, Sigma-Aldrich) and diluted 1:4000. Membranes were then washed with PBS-T and incubated with a horseradish peroxidase-conjugated goat anti-rabbit IgG antibody for $2 \mathrm{~h}$ at room temperature (1:10000 dilution). Antibody detection was performed with an enhanced chemiluminescence reaction (ECL western blotting detection; Amersham Life Science, UK).

\section{PCR}

\section{RNA extraction and reverse transcription}

From cultured cells, total RNA isolation was obtained after using the NucleoSpin RNA II Kit (Macherey-Nagel), allowing the purification of $\sim 5 \times 10^{6}$ cultured cells. Final RNA was dissolved in RNase-free water. The purity and quality of the
RNA purificated were also checked. Reverse transcription with elimination of genomic DNA was carried out according to the manufacture's instructions (QuantiTect Reverse transcription Handbook, Qiagen). We carried out all the reactions on ice to minimize the risk of RNA degradation. The cDNA obtained was kept at $-80^{\circ} \mathrm{C}$.

\section{Amplification}

From the cDNA preparation, $4 \mu \mathrm{l}$ was used in PCR with specifics primers (according to the modified method of Bigioni et $a l^{31}$ based on the common sequence of the TAC1 human isoforms (NM 001058, NM 015727) TAC1-F (CTGCTGGTGATTGGCTATGC) and TAC1-R (AGGAGGAAGAAGATGTGGAAGG), which yielded a $186 \mathrm{bp}$ fragment. The amplification of the specimen was performed in a final reaction volume of $20 \mu \mathrm{l}$, and was incubated at $95^{\circ} \mathrm{C}$ for $7 \mathrm{~min}$, subjected to 40 cycles of $95^{\circ} \mathrm{C}$ for $30 \mathrm{~s}, 62^{\circ} \mathrm{C}$ for $40 \mathrm{~s}$ and $72^{\circ} \mathrm{C} 30 \mathrm{~s}$, followed by a final extension cycle at $72^{\circ} \mathrm{C}$ for $7 \mathrm{~min}$. The amplification products were visualized by electrophoresis on $2 \%$ agarose gel stained with ethidium bromide.

\section{Immunohistochemistry for the NK-1 Receptor Patients}

In all, 37 human malignant melanoma specimens were retrieved from the archives of the Department of Pathology of the Virgen del Rocío University Hospital (Sevilla, Spain). Of these, 27 were primary invasive melanomas of the skin and 10 were metastatic melanomas. All melanomas had at least one focus of level IV invasion according to the Clark system. ${ }^{32}$ The thickness of the tumor was evaluated according to the Breslow system. ${ }^{33}$ The experimental design, protocols and procedures of this study were performed under the guidelines of the ethics and legal recommendations of Spanish and European law, as well as in accordance with the Declaration of Helsinki.

\section{Methods}

Paraffin-embedded human malignant melanoma tissues cut at $5 \mu \mathrm{m}$ and dried overnight at $60^{\circ} \mathrm{C}$ were used. As previously reported by us, ${ }^{16}$ to determine the specificity of the immunostaining, human gliomas were used as a positive control, whereas as a negative control the primary antibody was omitted, being replaced by nonimmune serum, and in both cases, the results obtained confirmed the specificity of the anti-NK-1 receptor antibody (Product number S8305, Sigma-Aldrich) used. Moreover, the anti-NK-1 receptor was preabsorbed ( $100 \mu \mathrm{g}$ per $\mathrm{ml}$ of diluted antiserum) with the synthetic peptide KTMTESSSFYSNMLA (synthesized by GenWay Biotech, San Diego, USA; see western blot analyses) and after the immunocytochemical protocol, no immunostaining appeared. All slides were evaluated by three independent pathologists. In all, 10 representative highpower microscopic fields were evaluated on each slide using a $\times 40$ objective. The presence or absence of staining and the 
intensity of the immunoreactivity were noted, as well as the number of cells showing a brown staining and whether the staining was localized in the tumor cells and/or in the plasma membranes. Tumors were recorded as positive when they showed cellular and/or plasma membrane staining ranging from moderate to strong in $>10 \%$ of the tumor cells. By consensus among the pathologists, the intensity of the immunoreactivity was evaluated as 'acceptable' only if the tumor cells showed a clear brown staining localized in the cytoplasm and/or plasma membrane of the tumor cells. The number of 'acceptable' immunoreactive cells was quantified as follows: negative $(0)$ if the immunoreactivity was observed in $<10 \%$ of the tumor cells; positive $(+)$ if the immunoreactivity was found to be between 11 and $50 \%$ of the tumor cells; and very positive $(++)$ if it was present in $>50 \%$ of the tumor cells (see Table 1).

\section{Small Interfering RNA Gene Silencing Method}

We carried out this method according to the manufacturer's instructions (Invitrogen, Madrid, Spain) and according to a previous study. ${ }^{34}$ One day before transfection, $2 \times 10^{4}$ MEL HO human melanoma cells per well were seeded in 6-well plates containing $2 \mathrm{ml}$ of normal growth medium. Total cell number was determined the day before the transfection. Cells were incubated at $37^{\circ} \mathrm{C}$ in a $\mathrm{CO}_{2}$ incubator for $17-20 \mathrm{~h}$ until $50-60 \%$ of the cells were confluent. The normal growth medium was then removed, and an antibiotic-free growth medium (Opti-MEM; Gibco) was added and incubated for $1 \mathrm{~h}$. The latter medium was removed and the transfection mixture was added, which contained the small interfering RNA (siRNA) TAC 1 (Invitrogen) and the diluted transfection reagent medium (Hiperfect, Qiagen); the two latter were previously incubated for $30 \mathrm{~min}$. Thereafter, the final volume obtained was $200 \mu \mathrm{l}$. In the same manner it was applied to siRNA-negative control solution. For each transfection, $200 \mu \mathrm{l}$ siRNA transfection reagent mixture was added to each well containing $800 \mu \mathrm{l}$ of the antibiotic-free growth medium. In all, $20 \mathrm{nM}$ was the final siRNA concentration used for each transfection. It was incubated for $5 \mathrm{~h}$ at $37^{\circ} \mathrm{C}$ in a $\mathrm{CO}_{2}$ incubator. Finally, $2 \mathrm{ml}$ of normal growth medium was added for additional $48-72 \mathrm{~h}$ incubation.

\section{RESULTS}

\section{NK-1 Receptors}

We carried out western blot analyses to test the presence of NK-1 receptors in MEL HO, COLO 858 and COLO 679 human melanoma cell lines. Incubation of the membranes with the anti-NK-1 receptor antibody (see the 'Materials and methods' section) showed the presence of different isoforms of the NK-1 receptor in human melanoma cell lines studied (Figure 1). Three similar bands (isoforms of $\sim 33,58$ and $75 \mathrm{kDa}$ ) were observed in the three melanoma cell lines studied (Figure 1b).

As a positive control, we included a protein extract from a glioma cell line. As previously reported ${ }^{10}$, we observed bands of 33, 37 and $75 \mathrm{kDa}$ (see Figure 1c). In addition, no band was detected when the incubation was carried out with the secondary antibody alone (see Figure 1a).

By PCR analyses, we also showed that MEL-HO, COLO 858 and COLO 679 tumor cell lines expressed mRNA for the tachykinin NK-1 receptor (Figure 2). NK-1 mRNA expression was detectable in these cell lines as a product of the expected size of $186 \mathrm{bp}$ corresponding to only one band, because the primers used in our PCR were designed for both short and long isoforms.

The knockdown method was carried out in two of the three melanoma cell lines studied. Thus, after $72 \mathrm{~h}$, we found $4 \times 10^{5}$ siRNA-negative control MEL HO cells and $1 \times 10^{5}$ siRNA TAC 1 cells (Figure 3a). Moreover, after the administration of siRNA TAC 1 to MEL HO cultured cells, many apoptotic cells were found in all the wells studied. In fact, in DAPI-stained cultures, we observed means of $40 \pm 1.4$ (s.d.)\% of apoptotic cells. However, in siRNA-negative control MEL-HO cells, we observed means of $5 \pm 0.4$ (s.d.)\%. With regard to COLO 679 melanoma cells, after $72 \mathrm{~h}$, we found $7.2 \times 10^{5}$ siRNA-negative control cells and $1.8 \times 10^{5}$ siRNA TAC 1 cells (Figure $3 \mathrm{~b}$ ). As before, after the administration of siRNA TAC 1 to COLO 679 cultured cells, many apoptotic cells were found. Thus, in DAPI-stained cultures, we observed means of $34 \pm 2.7$ (s.d.) $\%$ of apoptotic cells, but in siRNA-negative control COLO 679 cells, we observed means of $6 \pm 0.5$ (s.d.)\%. All these data show that NK-1 receptors have an important role in the viability of such tumor cells.

\section{NK-1 Receptors in Primary Invasive Malignant Melanoma of the Skin and Metastatic Melanoma}

Using an immunohistochemical technique, 37 human malignant melanomas were examined for the presence of NK-1 receptors. The expression of NK-1 receptors was observed in all the malignant melanoma samples studied (37/37), in both primary invasive malignant melanomas (27/27) and metastatic melanomas (10/10). Immunoreactivity was predominantly located in the cytoplasm of tumor cells (see Figures 4c and 5). Moreover, in three cases, ${ }^{19,20,26}$ an enhancement of the staining in the plasma membrane of these tumor cells was observed (Figure 4c). In seven of the primary invasive malignant skin melanomas (see Table 1), immunoreactivity for NK-1 receptors was observed in the smooth muscle cells of the small- and medium-caliber blood vessels, which were located in the peritumoral area, and occasionally, such immunoreactivity was also observed in the endothelial cells of these vessels. In 11 cases (see Table 1), immunoreactivity was observed in hair follicles, sebaceous glands or in sweat glands and in 17 of them, keratinocytes showed NK-1 immunoreactivity (see Table 1). Both follicles and glands showed cytoplasmic immunoreactivity, even in cases in which such structures were inside the tumor. Keratinocytes of the thorny and granular strata of the supratumoral epidermis showed an evident nuclear 
Table 1 Clinical findings: evaluation of the expression of the NK-1 receptor and its immunolocalization in tumor cells of primary invasive malignant melanomas of the skin and metastatic melanomas (M)

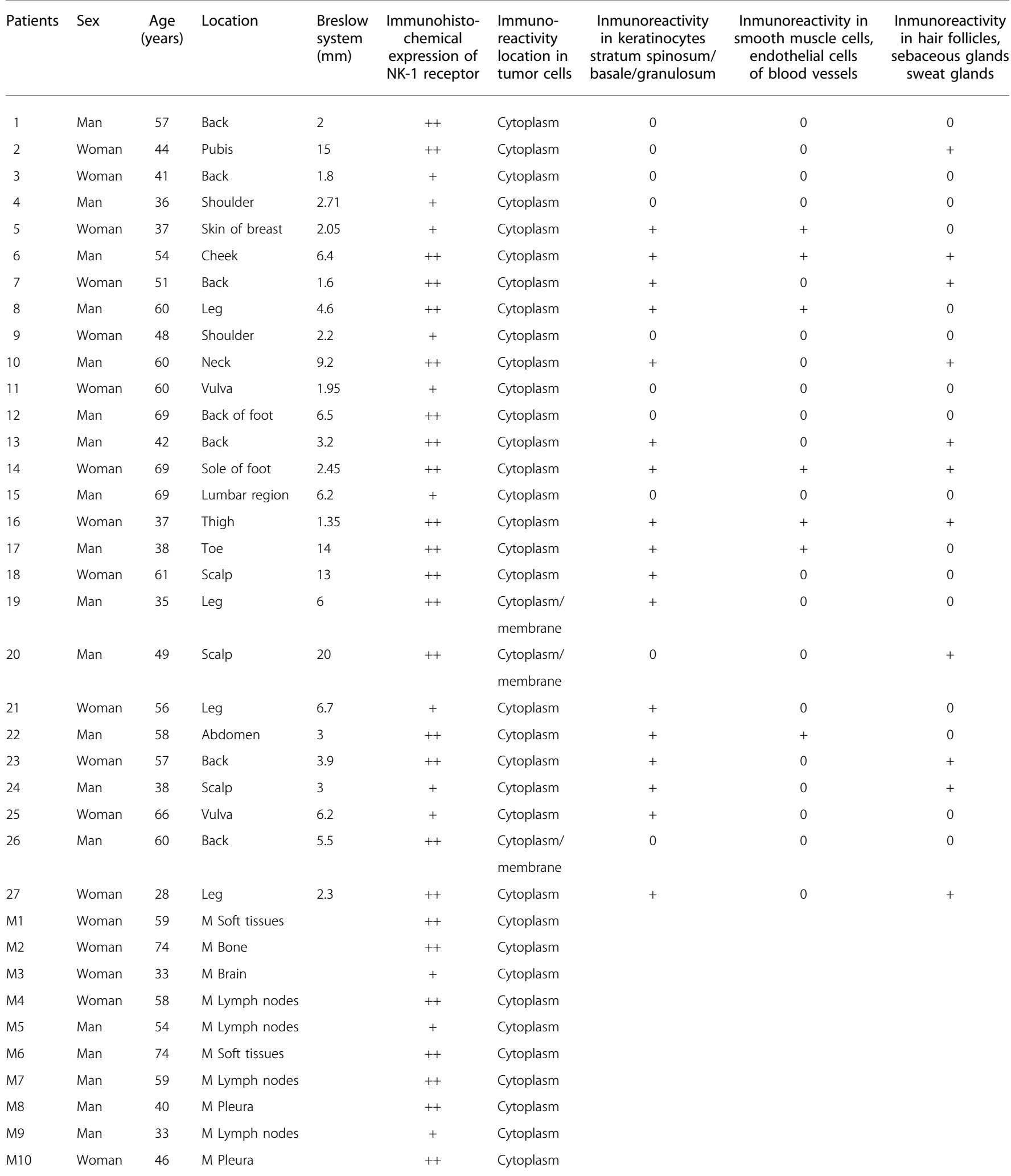

The expression of the NK-1 receptors is also evaluated in keratinocytes, smooth muscle cells, hair follicles and in sebaceous and sweat glands. 


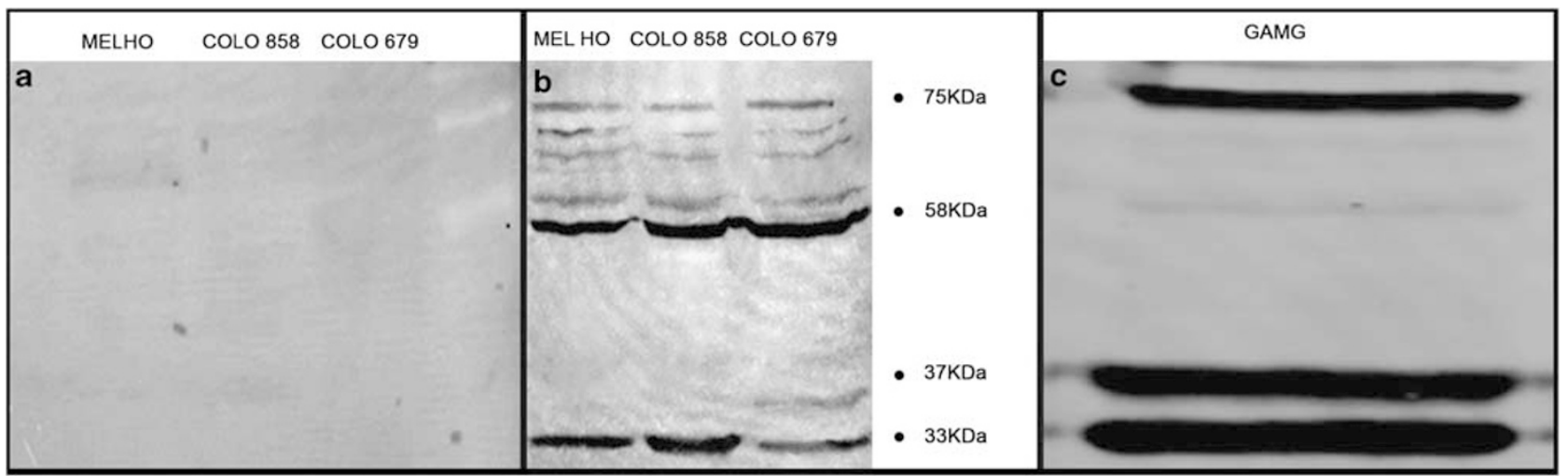

Figure 1 Western blot analysis of the NK-1 receptor in MEL HO, COLO 858 and COLO 679 human melanoma cell lines, showing the presence of different NK-1 receptor complex isoforms (b). The analysis was conducted three times. Dots indicate bands with molecular weights similar to those reported previously. (a) No band was detected when incubation was carried out with the secondary antibody alone. (c) Positive control: a protein extract from a glioma cell line (GAMG). ${ }^{10}$ In this cell line, bands of 33,37 and $75 \mathrm{kDa}$ were observed.

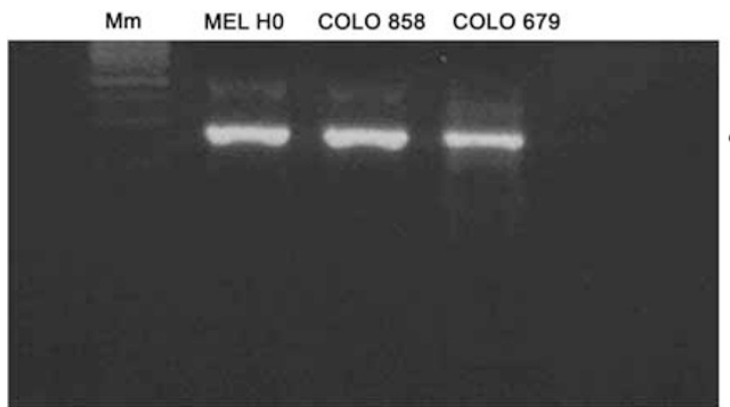

Figure 2 Agarose gel showing expression of TAC1-R CDNA in human melanoma cell lines. Equal aliquots of each cDNA were amplified by PCR for 40 cycles (target genes) with specific primers. PCR was carried out three times.

immunoreactivity. This suggests that the interior of the nucleus might have NK-1 receptors. In six cases of primary invasive malignant melanomas, the peritumoral area was not evaluated because the sample contained only tumor. The clinical data of the patients included in this study, together with the results of the evaluation of the expression of NK-1 receptors in tumor and no tumor cells and the location of such expression, are shown in Table 1 (also see Figures 4 and 5). Thus, for example, the expression of the NK-1 receptors was reported as positive (between 11 and $50 \%$ of the tumor cells showed immunoreactivity) and very positive $(>50 \%)$. This means that we observed immunoreactive and nonimmunoreactive cells in the same histological section (see Figure 5g, h).

\section{Antitumor Action of Aprepitant}

Growth inhibition of melanoma cell lines by aprepitant was observed after the addition of increasing concentrations of the drug (Figure 6a). Moreover, treatment of the three tumor cell lines with aprepitant resulted in concentration-dependent cytotoxicity (see Figure 6a). The $\mathrm{IC}_{50}$ and $\mathrm{IC}_{100}$ growth

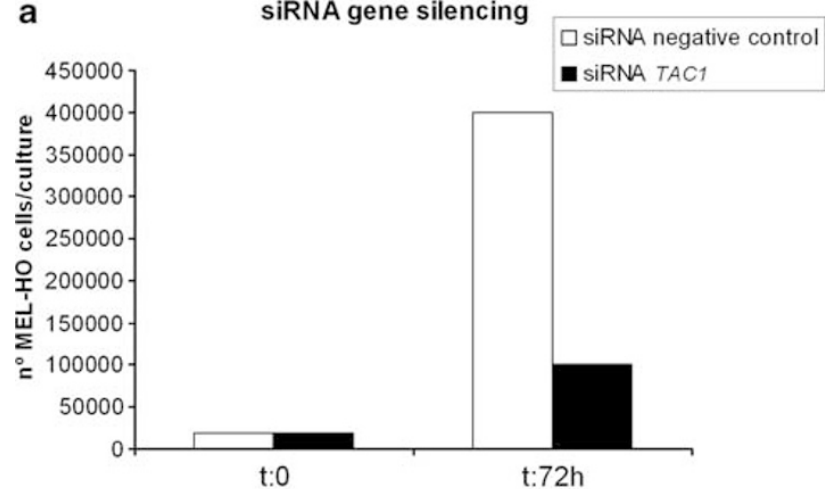

b siRNA gene silencing

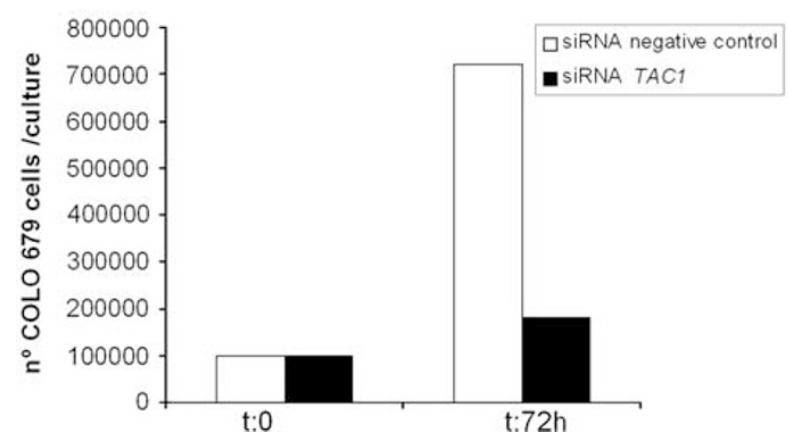

Figure 3 Viability of MEL HO and COLO 679 melanoma cells. This experiment was repeated three times in duplicate. The number of siRNAnegative control cells is compared with that of siRNA TAC 1 cells. At time 0 , 30000 MEL HO and 100000 COLO 679 cells were seeded. In both cases, at $72 \mathrm{~h}$, the decrease in the number of siRNA TAC 1 cells was quite significant in comparison with the number of siRNA-negative control cells.

inhibition aprepitant concentrations of melanoma cell lines are shown (in Table 2). Maximum inhibition was observed when the drug was present at a concentration of $\sim 60 \mu \mathrm{M}$ during the culture periods. At the first doubling time, a strong decrease in the number of melanoma cell lines studied 

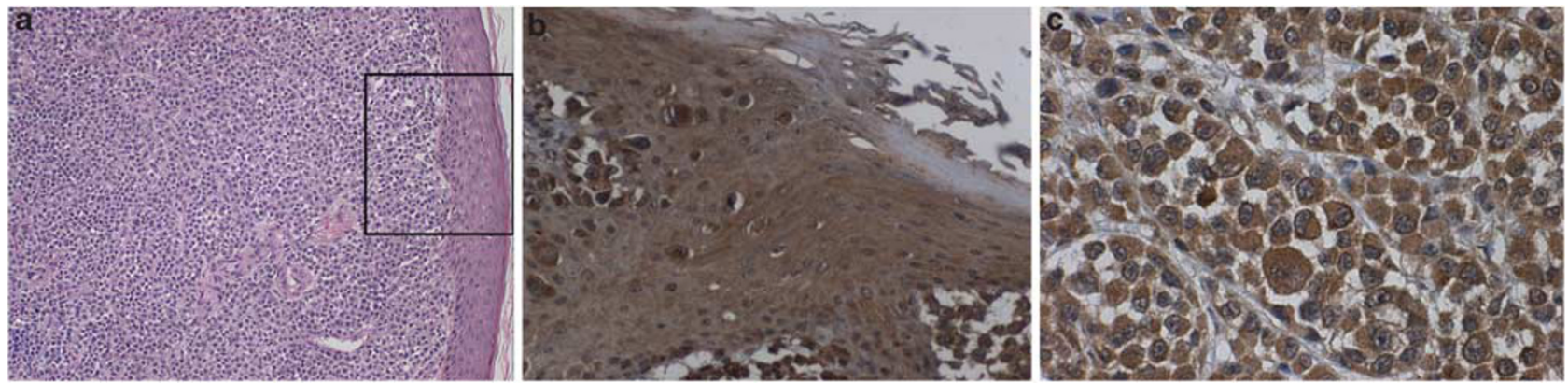

Figure 4 Case 20: (a) Primary malignant skin melanoma. Hematoxylin-eosin-stained: medium-power magnification. Epidermis and dermis can be observed in the delimited region. (b) No immunoreactivity for NK-1 receptors was observed in keratinocytes of the granular and thorny strata. (c) Strong immunoreactivity for NK-1 receptors was observed in tumor cells. Medium-power magnification. An enhancement of the staining in the plasma membrane of such cells is noted. The immunocytochemical technique was repeated at least three times.
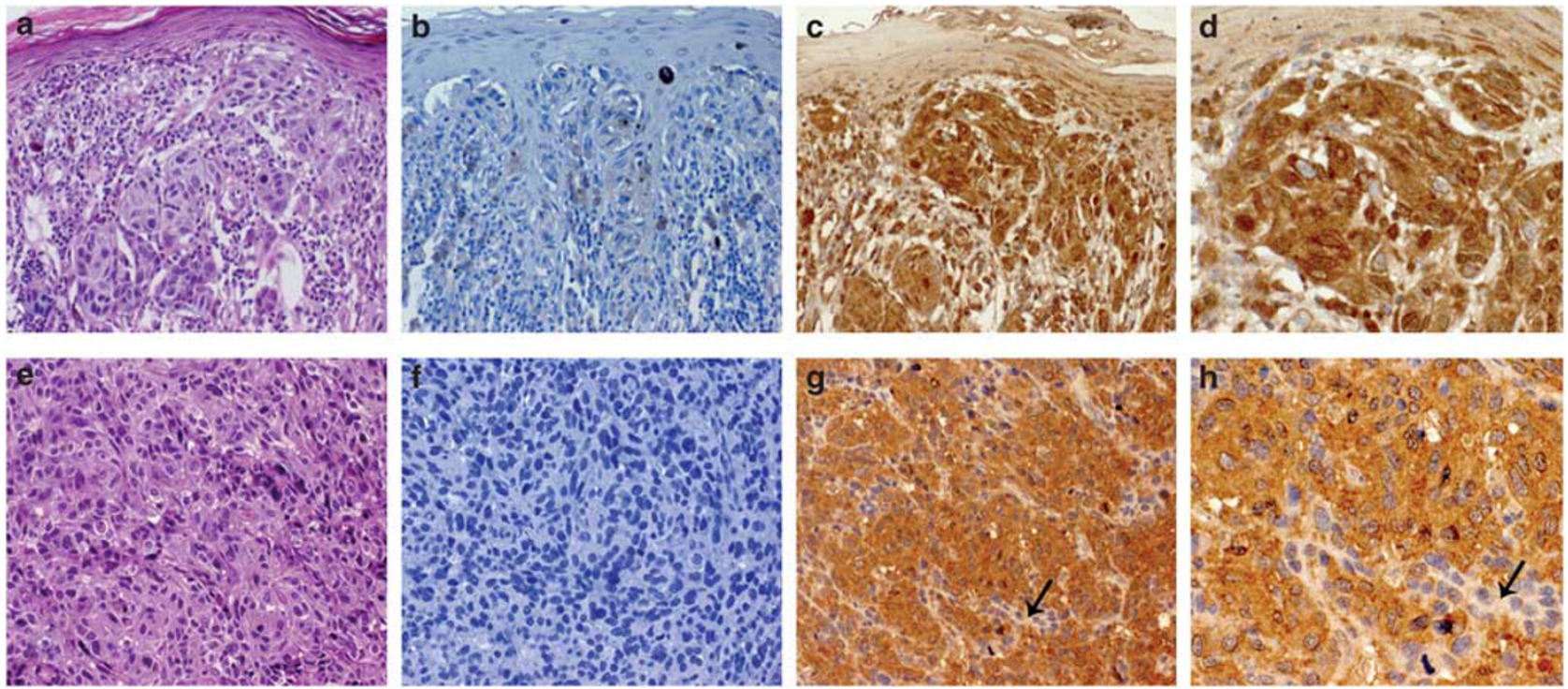

Figure 5 Case 16: (a) Primary malignant skin melanoma. Hematoxylin-eosin-stained: medium-power magnification. (b) Negative control: medium-power magnification. (c, d) Immunoreactivity located in tumor cells: medium- and high-power magnification, respectively. Case M1: (e) Metastatic soft tissue melanoma, Hematoxylin-eosin-stained: medium-power magnification. (f) Negative control: medium-power magnification. (g, h) Immunoreactivity located in tumor cells: medium- and high-power magnification, respectively. In panels $\mathrm{g}$ and $\mathrm{h}$, the presence of nonimmunoreactive cells (arrows) must be noted. In both 16 and $\mathrm{M} 1$ cases, the immunocytochemical technique was repeated at least three times.

was found at intermediate concentrations and no remaining living cells were observed at the maximum concentration. In all cell lines studied, a lower inhibition of growth was observed in the presence of low doses of aprepitant. There were no significant differences between the controls and the control-acetonitrile groups (data not shown).

\section{The NK-1 Receptor Antagonist Aprepitant Blocks SP-Induced Mitogen Stimulation}

Our group has previously reported that the three melanoma cell lines studied herein grew after the addition of different doses of SP. ${ }^{6}$ To show that the NK-1 receptor antagonist aprepitant inhibits melanoma cell proliferation by interaction with its receptor, we used the specific NK-1 receptor agonist $\mathrm{SP}$ in competition experiments. Thus, the cellular con- centration at the $\mu \mathrm{M} \mathrm{IC}_{50}$ of aprepitant and the most mitogenic nanomolar concentration of SP were lower than those observed after using different doses of SP alone for the three melanoma cell lines studied. These results indicate that aprepitant blocks SP mitogen stimulation, and that aprepitant-induced growth inhibition was partially reversed by the administration of the most mitogenic nanomolar dose of exogenous SP. In this sense, we observed higher cellular concentrations when compared with the data found after the administration of aprepitant alone (see Figure 7). This indicates that SP specifically activates NK-1 receptors during the growth of human melanoma cell lines.

Moreover, aprepitant-induced growth inhibition was partially reversed by the administration of gradual increasing concentrations of SP. The maximum effect was observed after 
a
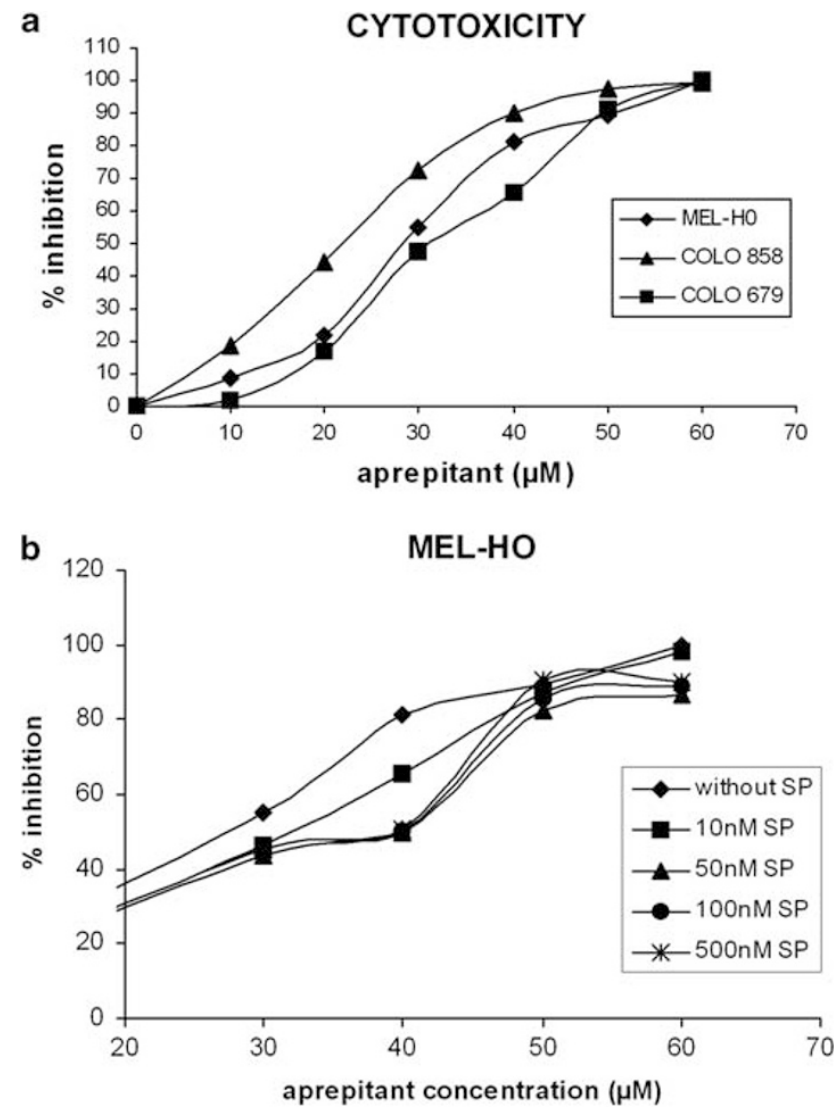

Figure 6 (a) Percentage of growth inhibition of MEL HO, COLO 858 and COLO 679 human melanoma cells at first doubling time in in vitro cultures after the addition of increasing concentrations (10-60 $\mu \mathrm{M})$ of aprepitant. Level of significance: ${ }^{*} P \leq 0.05$. The experiment was repeated three times in duplicate. (b) MEL HO human melanoma: aprepitant-induced growth inhibition was gradually reversed by the administration of gradual increasing concentrations of SP. Level of significance: ${ }^{\star *} P \leq 0.05$. The experiment was conducted three times in duplicate.

Table 2 Results of half-inhibition $\left(\mathrm{IC}_{50}\right)$ and maximum inhibition $\left(\mathrm{IC}_{100}\right)$ experiments in melanoma cells after administration of the NK-1 receptor antagonist aprepitant

Melanoma cell line

Aprepitant

$\overline{\mathrm{IC}_{50} \mu \mathrm{M} \quad \mathrm{IC}_{100} \mu \mathrm{M}}$

MEL HO

29.6

56.5

COLO 858

24.3

52.1

COLO 679

32.1

58.6

the administration of $50 \mathrm{nM}$ of exogenous SP, in competition with different concentrations of aprepitant (Figure 6b). This means that aprepitant does not exert an off-target effect on melanoma cell lines and that the antitumor action of aprepitant is mediated through NK-1 receptors.

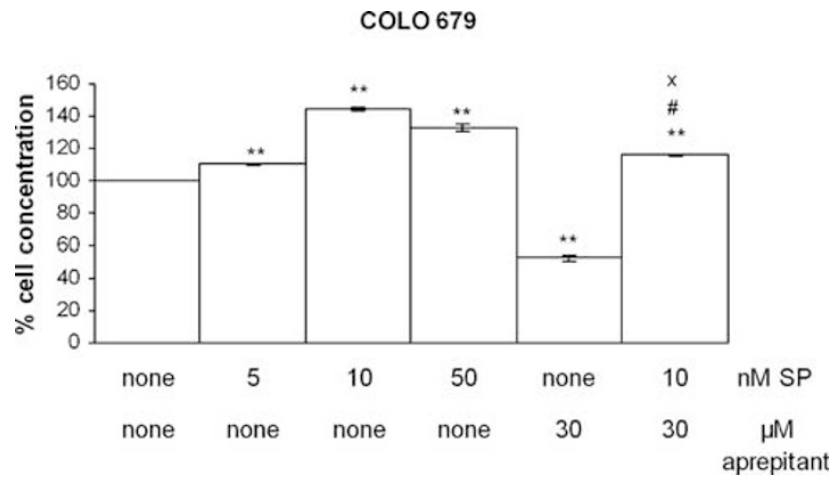

Figure 7 Induction of cell proliferation of COLO 679 human melanoma cells by SP at several nanomolar concentrations $(5,10$ and $50 \mathrm{nM})$. The experiment was conducted at least three times in duplicate. The NK-1 receptor antagonist aprepitant was added $(30 \mu \mathrm{M})$ in the presence $(10 \mathrm{nM})$ or absence (none) of SP for the first doubling time. In both cases, aprepitant inhibited COLO 679 cell proliferation. Using the ANOVA test, a significant difference between each group and the control group (none-none) was found. Level of significance: ${ }^{* *} P \leq 0.05$ and \# indicates the value of significance of 10 -none vs $10-30$ and $\times 10-30$ vs none -30 \# and $\times P \leq 0.05$. Vertical bars indicate s.d.

\section{Apoptosis}

After the administration of aprepitant, many apoptotic cells were found in all melanoma cell lines studied (Figure 8). In fact, in DAPI-stained cultures, we observed means of $28 \pm 8.1$ (s.d.)\% at the $\mathrm{IC}_{50}$ concentration and of $36 \pm 7.6$ (s.d.) $\%$ at the $\mathrm{IC}_{100}$ concentration of apoptotic cells in all melanoma cell lines studied after the administration of aprepitant. Conversely, in control melanoma cells (not treated with aprepitant), we observed means of $0.57 \pm 0.6$ (s.d.)\% of apoptotic cells in all three melanoma cell lines.

\section{DISCUSSION}

In this study, we showed for the first time the presence of NK-1 receptors in MEL HO, COLO 858 and COLO 679 human melanoma cell lines and the presence of NK-1 receptors in human primary invasive malignant melanomas and metastatic melanomas.

We carried out several controls to check the specificity of the anti-NK-1 receptor antibody used in this study: (1) omission of the primary antibody (immunohistochemistry and western blot); (2) preabsorption of the primary antibody with the corresponding synthetic peptide (immunohistochemistry); and (3) the use of human gliomas as positive controls (immunohistochemistry and western blot). In all the cases, the results confirmed the specificity of the anti-NK-1 receptor antibody used in this study. It should also be noted that in the same human melanoma sample, we observed cells expressing or not expressing NK-1 receptors. Moreover, after eliminating NK-1 receptors by a knockdown method, we showed that NK-1 receptors are involved in the viability of tumor cells, as well as that these two melanoma cell lines express mRNA for the NK-1 receptor. 

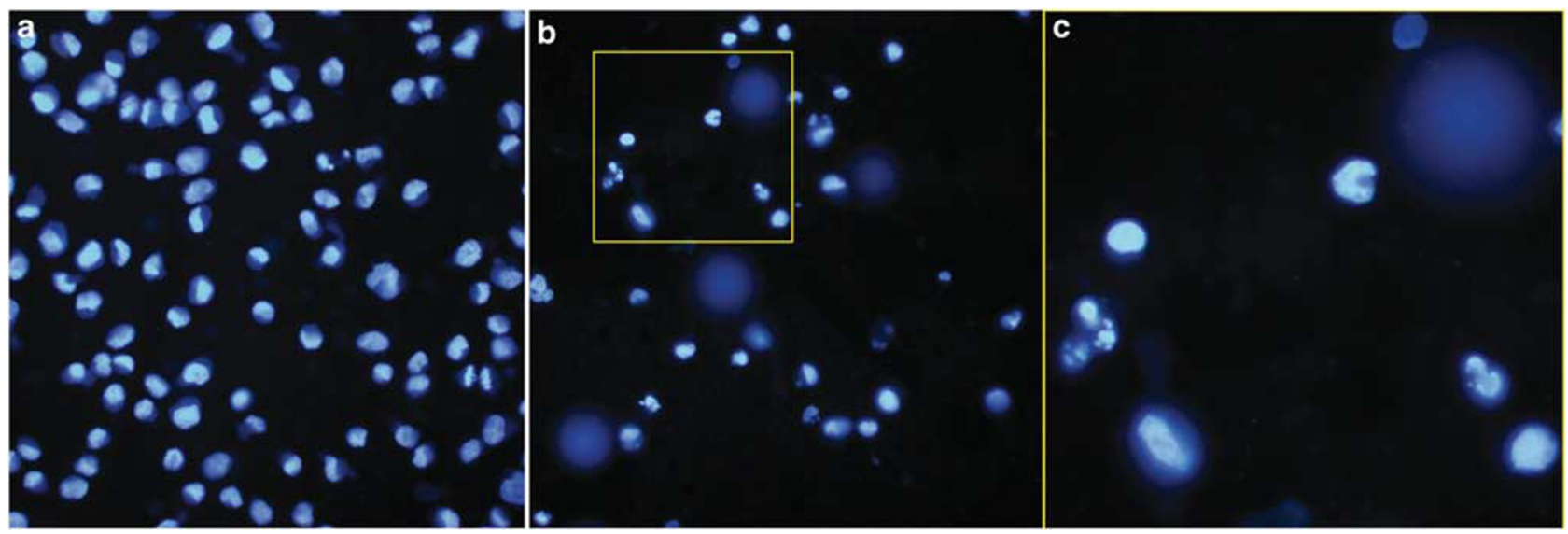

Figure 8 (a) Untreated COLO 858 melanoma cell culture. (b) COLO 858 melanoma cells treated with the NK-1 receptor antagonist aprepitant. The experiment was repeated three times. Apoptotic figures: the chromatin condensation and the nuclear fragmentation can be observed. (c) High-power magnification of the region delimited in panel $b$.

The data described herein are in agreement with those reported in previous studies, in which the presence of isoforms of the NK-1 receptor in neuroblastoma, glioma, retinoblastoma, pancreatic, larynx, colon and gastric carcinoma cell lines was reported. ${ }^{10,12,15,16,24}$ We show the presence of several NK-1 receptor isoforms $(33,58$ and $75 \mathrm{kDa})$ in MEL HO, COLO 858 and COLO 679 human malignant melanoma cell lines, although the functional roles of these NK-1 receptor isoforms are currently unknown. Until now, the expression of NK-1 receptors in human primary invasive malignant melanomas and metastatic melanomas was unknown. In this study, for the first time, we show the presence of NK-1 receptors in both cases. Our data are in agreement with those of previous studies, as it is known that NK-1 receptors are overexpressed in primary glioblastomas, breast cancer and its metastasis, pancreatic carcinoma, retinoblastoma, and larynx, gastric and colon carcinomas. ${ }^{16,19-22,24}$ Moreover, it has been shown that NK-1 receptor expression is increased 25-36-fold in human pancreatic cancer cell lines in comparison with normal controls, and that tumors samples obtained from patients with advanced tumor stages exhibit significantly higher NK-1 receptor levels. ${ }^{21}$ Thus, using immunohistochemical methods, NK-1 receptor visualization should facilitate the identification of tumors with a sufficient NK-1 receptor overexpression for diagnostic and therapeutic intervention (administering NK-1 receptor antagonists)., ${ }^{4,23}$ It is known that the activation of the NK-1 receptor by SP at nanomolar concentrations induces mitogenesis in human malignant melanoma cell lines ${ }^{6}$ and in several cancer cell lines, ${ }^{8,10-13,15,16}$ and also that SP is expressed in primary invasive malignant melanomas and metastatic melanomas. ${ }^{5}$

We showed that treatment of the three melanoma cell lines studied herein with the NK-1 receptor antagonist aprepitant causes growth inhibition and cell death. We also showed that the cell death observed in this study was due to a specific toxic effect of aprepitant and not a nonspecific action of this drug. In our competition experiments, exogenous SP cell proliferation was partially reversed by the administration of aprepitant in the three melanoma cell lines, indicating the specificity of NK-1 receptor blockade by aprepitant. Moreover, aprepitant-induced growth inhibition was gradually reversed by gradual increasing concentrations of SP. Thus, the antitumor action on these melanoma cell lines by aprepitant is probably related to the ability of this antagonist to block the NK-1 receptor. In addition, the findings of this study show that treatment of the three melanoma cell lines with this NK-1 receptor antagonist results in cell death and that such death occurs by apoptosis. This is in agreement with previous in vitro studies carried out in lung cancer, ${ }^{35}$ rhabdomyosarcoma, ${ }^{7}$ neuroblastoma, retinoblastoma, and larynx, gastric and colon carcinoma cell lines. ${ }^{14-16,24}$

In a previous study, it has been reported that NK-1 receptors mediate shape changes in normal human embryonic kidney cells (HEK 293) and that these changes were prevented after preincubation with NK-1 receptor antagonists at $1 \mu \mathrm{M}$ concentration. ${ }^{36}$ We have previously reported that the $\mathrm{IC}_{50}$ for the NK-1 receptor antagonist aprepitant against HEK 293 cells, was $>90 \mu \mathrm{M}$ (30). At $1 \mu \mathrm{M}$ of aprepitant, we did not observe any antitumoral action on the tumor cells studied herein. On the contrary, we observed such action when the concentration of aprepitant was increased. This could be explained because tumor cells overexpress NK-1 receptors, ${ }^{4}$ and for this reason, it is necessary to increase the concentration of NK-1 receptor antagonists to observe an antitumoral action on cancer cells. Previously, the effect of SP on the growth of capillary vessels in vivo and on the proliferation of cultured endothelial cells in vitro has been reported. In this sense, neoangiogenesis, probably due by endothelial cell proliferation, could be blocked specifically by NK-1 receptor antagonists. ${ }^{17,37}$ Furthermore, neoangiogenesis is a sequential process with early endothelial proliferation, followed by vessel neoformation and increased blood 
flow; the maturation of endogenous neurovascular regulatory systems occurs late in this process in inflamed tissues. ${ }^{38,39}$ Vessel neoformation has also been associated with increased tissue innervation and expression of NK-1 receptors; ${ }^{20}$ indeed, in a large majority of the tumors investigated, NK-1 receptors have been found on intratumoral and peritumoral blood vessels ${ }^{19}$ and this finding is in agreement with the results reported herein. In addition, both SP and histamine (released from mast cells by the action of SP) are often concentrated in the connective tissue surrounding many tumors, including melanomas, and become so before the development of neoangiogenesis. ${ }^{5}$ The findings reported in this study show that the expression of the NK-1 receptor in tumor vessels is consistent with the above studies. Conversely, $\mathrm{SP}$ analog antagonists, synonymous of NK-1 receptor antagonists, inhibit tumor growth in pancreatic cancer by a dual mechanism involving both antiproliferative and antiangiogenic properties. ${ }^{40}$ The active migration of tumor cells is a crucial requirement for the development of metastasis and cancer progression. It is known that SP induces the migration of tumor cells to specific organs by binding to NK-1 receptors in cancer cells, where it can be blocked by NK-1 receptor antagonists. ${ }^{18}$ Moreover, a recent study carried out in HEK 293 cells has reported that the activation of the NK-1 receptor by SP induced a rapid cellular shape change, including blebbing. This is not associated with apoptosis, but it is a consequence of the Rho-activated ROCK system, leading to an increase in the phosphorylation of the myosin regulatory light chain. ${ }^{36}$ Membrane blebbing is important in cell movement, cell spreading and in cancer cell invasion. ${ }^{41}$ The same study reported that aprepitant and L-733060 blocked SP-induced rapid cellular shape change, including blebbing. ${ }^{36}$

These data suggest that SP and NK-1 receptors could have an important role in the development of invasion and metastasis. In addition, some alarming signs of the malignization of the nevi are pain, pruritus, inflammation and increased size. These signs could be due to an increase in SP in the perilesional zone of the skin produced by the different mechanisms involved in neurogenic inflammation. In fact, the release of SP from peripheral sensory fibers could produce vasodilatation, increase vascular permeability and plasma extravasation, as well as induce the release of histamine (pruritus) from skin mast cells. In addition, melanoma cells releasing SP could cause tumor proliferation.

All the data mentioned above suggest that the use of NK-1 receptor antagonist aprepitant could improve melanoma treatment because the drug could exert an antitumoral action through three mechanisms: (1) an antiproliferative effect, due to the inhibition of tumor cell growth, inducing cell death by apoptosis; (2) an inhibition of angiogenesis in the tumor mass; and (3) an inhibition of the migration of tumor cells (invasion and metastasis). Moreover, aprepitant has also been used in a placebo-controlled trial in patients with depression, with the observation that the safety and the tolerability of this drug are generally similar to the placebo. ${ }^{27}$ Recently, we have reported the safety of aprepitant against human fibroblast cells (HEK 293). Thus, the IC $_{50}$ for HEK 293 fibroblast cells was approximately three times higher than the $\mathrm{IC}_{50}$ for tumor cells, ${ }^{30}$ as occurring in the melanoma cell lines studied here. Indeed, in the host, aprepitant exerts beneficial effects in a dose-dependent manner, such as antiemetic, ${ }^{29}$ antidepressant, ${ }^{27}$ anxiolytic, ${ }^{28}$ anti-inflammatory, ${ }^{42}$ analgesic, ${ }^{43}$ hepatoprotector ${ }^{42}$ and neuroprotector. ${ }^{44}$

In summary, we describe for the first time the presence of several isoforms of the NK-1 receptor in MEL HO, COLO 858 and COLO 679 human malignant melanoma cell lines. We identified the presence of NK-1 receptors in all human primary invasive malignant melanomas and metastatic melanomas studied, and we report the antitumoral action of the NK-1 receptor antagonist aprepitant on human melanoma cell lines. We also show that the antitumoral action of aprepitant is through the NK-1 receptor and that aprepitant induces apoptosis in melanoma cell lines. Finally, we have also reported that NK-1 receptors have an important role in the viability of tumor cells and that the above-mentioned melanoma cell lines express mRNA for the NK-1 receptor. All these observations suggest that the NK-1 receptor is a new candidate target in the treatment of human melanoma and that the NK-1 receptor antagonist aprepitant could be a novel and promising antitumor drug in the treatment of melanoma.

\section{ACKNOWLEDGEMENTS}

We thank Dr José Palacios (Department of Pathology, 'Virgen del Rocío' University Hospital, Sevilla, Spain) for providing human malignant melanomas, Dr Andrés Carranza for his help, Mr Manuel Sánchez and Francisco Jesús Fuentes for technical assistance, Mr Nicholas Skinner (University of Salamanca, Spain) for supervising the English text and Professor Amancio Carnero (Spanish National Cancer Center, Madrid) for supervising the knockdown method. This work was supported by the Consejería de Innovacion, Ciencia y Empresa of the Junta de Andalucía (CTS-2247, Spain).

\section{DISCLOSURE/CONFLICT OF INTEREST}

USPTO Application no. 20090012086 'Use of non-peptidic NK-1 receptor antagonists for the production of apoptosis in tumor cells'.

1. American Cancer Society. Cancer Facts and Figures 2007-2008. American Cancer Society: Atlanta, 2007.

2. Hökfelt $T$, Pernow B, Wahren J. Substance P: a pioneer amongst neuropeptides. J Intern Med 2001;249:27-40.

3. Harrison S, Geppetti P. Substance P. Int J Biochem Cell Biol 2001 ;33:555-576.

4. Muñoz $M$, Rosso $M$, Robles-Frías $M J$, et al. Immunolocalization of the neurokinin-1 receptor: a new target in the treatment of the human primary retinoblastoma. In: Bospene EB (ed). Focus on Eye Neoplasma Research Advances. Nova Science Publishers: New York, 2008, pp 157-178.

5. Khare VK, Albino AP, Reed JA. The neuropeptide/mast cell secretagogue substance $P$ is expressed in cutaneus melanocytic lesions. J Cutan Pathol 1998;25:2-10.

6. Muñoz $M$, Pérez $A$, Rosso $M$, et al. Antitumoural action of NK1 receptor antagonist L-733 060 on human melanoma cell lines. Melanoma Res 2004; 14:183-188.

7. Esteban $F$, Muñoz $M$, González-Moles $M A$, et al. A role for substance $P$ in cancer promotion and progression: a mechanism to counteract 
intracellular death signals following oncogene activation or DNA damage. Cancer Metast Rev 2006;25:137-145.

8. Luo W, Sharif TR, Sharif M. Substance P-induced mitogenesis in human astrocytoma cells correlates with activation of the mitogen-activated protein kinase signaling pathway. Cancer Res 1995;56:4983-4991.

9. Palma C, Nardelli F, Manzini $S$, et al. Substance $P$ activates responses correlated with tumor growth in human glioma cells line bearing tachykinin NK1 receptors. Br J Cancer 1999;79:236-243.

10. Muñoz M, Rosso M, Pérez A, et al. The NK1 receptor is involved in the antitumoural action of L-733060 and in the mitogenic action of substance $P$ on neuroblastoma and glioma cell lines. Neuropeptides 2005:39:427-432.

11. Muñoz M, Rosso $M$, Pérez $A$, et al. Antitumoural action of the neurokinin-1- receptor antagonist L-733060 and mitogenic action of substance $\mathrm{P}$ on human retinoblastoma cell lines. Invest Ophthalmol Vis Sci 2005:46:2567-2570.

12. Muñoz $M$, Rosso $M$, Coveñas $R$. The NK-1 receptor is involved in the antitumoural action of L-733060 and in the mitogenic action of substance P on human pancreatic cancer cell lines. Lett Drug Des Discovery 2006;3:323-329.

13. Muñoz M, Rosso M, Soult JA, et al. Antitumoural action of neurokinin-1 receptor antagonists on human brain cancer cell lines. In: Yang AV (ed). Brain Cancer: Therapy and Surgical Intervention. Nova Science Publishers: New York, 2006, pp 45-75.

14. Muñoz $M$, Rosso $M$, Coveñas R. NK-1 receptor antagonists as new anti-tumoural agents: action on human neuroblastoma cell lines. In: Fernandes JA (ed). Focus on Neuroblastoma Research. Nova Science Publishers: New York, 2007, pp 31-56.

15. Muñoz M, Rosso M, Aguilar FJ, et al. NK-1 receptor antagonists induce apoptosis and counteract substance P-related mitogenesis in human laryngeal cancer cell line HEp-2. Investig New Drugs 2008;26:111-118.

16. Rosso M, Robles-Frías MJ, Coveñas R, et al. The NK-1 receptor is expressed in human primary gastric and colon adenocarcinomas and is involved in the antitumor action of L-733060 and the mitogenic action of substance $\mathrm{P}$ on human gastrointestinal cancer cell lines. Tumor Biol 2008;29:245-254.

17. Ziche $M$, Morbidelli $L$, Pacini $M$, et al. Substance $P$ stimulates neovascularization in vivo and proliferation of cultured endothelial cells. Microvasc Res 1990;40:264-278.

18. Lang K, Drell TL, Lindecke A, et al. Induction of a metastatogenic tumor cell type by neurotransmitters and its pharmacological inhibition by established drugs. Int J Cancer 2004;112:231-238.

19. Hennig IM, Laissue JA, Horisberger U, et al. Substance-P receptors in human primary neoplasms: tumoral and vascular localization. Int J Cancer 1995;61:786-792.

20. Singh $D$, Joshi DD, Hameed $M$, et al. Increased expression of preprotachykinin-I and neurokinin receptors in human breast cancer cells: implications for bone marrow metastasis. Proc Natl Acad Sci USA 2000;97:1388-1393.

21. Friess $H, Z$ Zhu Z, Liard V, et al. Neurokinin-1 receptor expression and its potential effects on tumor growth in human pancreatic cancer. Lab Invest 2003;83:731-742.

22. Esteban F, González-Moles MA, Castro D, et al. Expression of substance $P$ and neurokinin-1-receptor in laryngeal cancer: linking chronic inflammation to cancer promotion and progression. Histopathology 2008;4:258-260; in press DOI: 10.1111/j.1365-2559.2008.03193.x.

23. Schulz S, Stumm R, Röcken C, et al. Immunolocalization of full-length $\mathrm{NK}_{1}$ tachykinin receptors in human tumors. J Histochem Cytochem 2006;54:1015-1020.

24. Muñoz $M$, Rosso $M$, Coveñas $R$, et al. Neurokinin-1 receptors located in human retinoblastoma cell lines: antitumor action of its antagonist, L-732 138. Invest Ophthalmol Vis Sci 2007;48:2775-2781.
25. Harrison T, Willians BJ, Swain CJ, et al. Piperidine-ether based hNK1 antagonists 1: determination of the relative and absolute stereochemical requirements. Bioorg Med Chem Lett 1994;4: 2545-2550.

26. Muñoz M, Pérez A, Coveñas R, et al. Antitumoural action of L-733 060 on neuroblastoma and glioma cell lines. Arch Ital Biol 2004;142: 105-112.

27. Kramer MS, Cutler N, Feighner J, et al. Distinct mechanism for antidepressant activity by blockade of central substance $\mathrm{P}$ receptors. Science 1998;81:1640-1645.

28. Varty GB, Cohen-Williams ME, Morgan CA, et al. The gerbil elevated plus-maze II: anxiolytic-like effects of selective neurokinin NK1 receptor antagonists. Neuropsychopharmacology 2002;27:371-379.

29. Tattersall FD, Rycroft W, Cumberbatch $M$, et al. The novel NK-1 receptor antagonist MK-0869 (L-754 030) and its water soluble phosphoryl prodrug, L-758 298, inhibit acute and delayed cisplatininduced emesis in ferrets. Neuropharmacology 2000;39:652-663.

30. Muñoz M, Rosso M. The NK-1 receptor antagonist aprepitant as a broad spectrum antitumor drug. Invest New Drugs 2009;28:187-193; in press DOI: 10.1007/s10637-009-9218-8 A.

31. Bigioni M, Benzo A, Irrissuto C, et al. Role of NK-1 and NK-2 tachykinin receptor antagonism on the growth of human breast carcinoma cell line MDA-MB-231. Anticancer Drugs 2005;16:1083-1089.

32. Clark Jr WH, From L, Bernardino EA, et al. The histogenesis and biologic behavior of primary human malignant melanomas of the skin. Cancer Res 1969;29:705-727.

33. Breslow A. Thickness, cross-sectional areas and depth of invasion in the prognosis of cutaneous melanomas. Ann Surg 1970;172:902-908.

34. Moneo V, Serelde BG, Leal JFM, et al. Levels of p $27^{\text {kip } 1}$ determine Aplidin sensitivity. Mol Cancer Ther 2007;6:1310-1316.

35. Reeve JG, Bleehen NM. [D-Arg ${ }^{1}-D-P h e^{5}$, D-Trp ${ }^{7,9}$, Leu ${ }^{11}$ ] Substance P induces apoptosis in lung cancer cell lines in vitro. Biochem Bioph Res Com 1994;199:1313-1319.

36. Meshki J, Douglas SD, Lai JP, et al. Neurokinin 1 receptor mediates membrane blebbing in HEK293 cells through a Rho/Rho-associated coiled-coil kinase-dependent mechanism. J Biol Chem 2009;284: 9280-9289.

37. Fan TP, Hu DE, Guard $\mathrm{S}$, et al. Stimulation of angiogenesis by substance $P$ an interleukin-1 in the rat and its inhibition by NK1 or interleukin-1 receptor antagonists. Br J Pharmacol 1993;110:43-49.

38. Walsh DA, Hu DE, Mapp Pl, et al. Innervation and neurokinin receptors during angiogenesis in the rat sponge granuloma. J Histochem Cytochem 1996;28:759-769.

39. Seegers $\mathrm{HC}$, Hood VC, Kidd BL, et al. Enhancement of angiogenesis by endogenous substance $P$ release and neurokinin-1 receptors during neurogenic inflammation. J Pharmacol Exp Ther 2003;306:8-12.

40. Guha S, Eibl G, Kisfalvi K, et al. Broad-spectrum G protein-coupled receptor antagonist, [D-Arg1,DTrp5,7,9,Leu11]SP: a dual inhibitor of growth and angiogenesis in pancreatic cancer. Cancer Res 2005;65:2738-2745.

41. Fackler OT, Grosse R. Cell motility through plasma membrane blebbing. J Cell Biol 2008;181:879-884.

42. Bang R, Sass G, Kiemer AK, et al. Neurokinin-1 receptor antagonists CP-96 345 and L-733 060 protect mice from cytokine-mediated liver injury. J Pharmacol Exp Ther 2003;305:31-39.

43. Rupniak NM, Carlson E, Boyce S, et al. Enantioselective inhibition of the formalin paw late phase by the NK1 receptor antagonist L- 733060 in gerbils. Pain 1996;67:189-195.

44. Yu J, Cadet JL, Angulo JA. Neurokinin-1 (NK-1) receptor antagonists abrogate methamphetamine-induced striatal dopaminergic neurotoxicity in the murine brain. J Neurochem 2002;83: 613-622. 